\title{
Éducation ou barbarie. Pour une anthropo-pédagogie contemporaine
}

Bernard Charlot, Economica Anthropos, 2020, 336 p.

Roger-François Gauthier

\section{CpenEdition}

\section{Journals}

Édition électronique

URL : https://journals.openedition.org/ries/10703

DOI : $10.4000 /$ ries. 10703

ISSN : 2261-4265

Éditeur

France Education international

Édition imprimée

Date de publication : 1 septembre 2021

ISBN : 978-2-85420-630-2

ISSN : $1254-4590$

Référence électronique

Roger-François Gauthier, "Éducation ou barbarie. Pour une anthropo-pédagogie contemporaine », Revue internationale d'éducation de Sèvres [En ligne], 87 | septembre 2021, mis en ligne le 01 septembre 2022, consulté le 26 mars 2023. URL : http://journals.openedition.org/ries/10703 ; DOI : https://doi.org/ 10.4000/ries. 10703

Ce document a été généré automatiquement le 26 mars 2023.

Tous droits réservés 


\section{Éducation ou barbarie. Pour une anthropo-pédagogie contemporaine}

Bernard Charlot, Economica Anthropos, 2020, 336 p.

Roger-François Gauthier

\section{RÉFÉRENCE}

Éducation ou barbarie. Pour une anthropo-pédagogie contemporaine, Bernard Charlot, Economica Anthropos, 2020, $336 \mathrm{p}$.

1 Bernard Charlot procède dans cet ouvrage comme Diogène qui parcourait Athènes, on le sait, avec sa lanterne, à la recherche de l'homme: lui parcourt l'histoire de l'éducation jusqu'à la période la plus contemporaine, mais aussi les thèses des anthropologues à la recherche de ce qui a pu ou pourrait fonder un projet d'éducation indiscutable, solide, qui ne soit bien sûr pas fondé sur la référence à une croyance, mais sur aucune autre chose que ce qu'on peut savoir de Sapiens, et de la fonction, pour lui, de l'éducation.

2 Reprenant par le menu les différentes théories qui ont sous-tendu les pédagogies depuis Platon jusqu'au christianisme, puis à la pensée rationaliste et républicaine, et même aux "pédagogies nouvelles", l'auteur observe qu'elles ont en commun que l'« éducateur incarne toujours un principe suprême qui justifie sa pédagogie » : le Salut de l'âme, la Raison, la République, ou la Nature, et que « ce qui justifie la discipline aux yeux de l'éducateur, c'est la désirabilité de la Sagesse, de la Sainteté, de la Citoyenneté ou du Savoir ». Or précisément, l'échec de toutes ces pédagogies est de n'être pas parvenues «à régler de façon durable et satisfaisante le rapport entre le désir et la norme ».

Voulant décrire ce qui caractérise selon lui la façon dont l'éducation est traitée dans le monde contemporain, il rappelle que jusqu'à une période récente, l'éducation était entourée partout d'un discours d'humanité, caractéristique de l'après-guerre, et 
qu'« on pouvait croire que la barbarie reculait dans le monde, grâce, notamment, à l'éducation ».

4 Or aujourd'hui, poursuit-il, changement majeur: « les discours qui dominent la scène de l'éducation focalisent l'efficacité et la performance ", alors que d'autres, "plus ou moins fanatiques, inspirés par la conviction religieuse, nationaliste, raciste, cherchent à imposer une hiérarchie de l'humain, à partir des critères traditionnels de domination, ou comme une contre-hiérarchie produite par ceux qui ont été victimes de discriminations». Alors même qu'«on n'entend plus de grands discours pédagogiques ».

Or précisément, poursuit-il, reprenant son idée que toute proposition d'éducation doit résoudre la confrontation entre une norme et le désir, la valorisation contemporaine du désir n'étant pas le fruit d'une conversion morale, mais résultant de l'évolution socio-économique, la norme qui fait face à cette extension du désir, c'est l'évaluation, la notation, la hiérarchisation. Ce qui gouverne l'école aujourd'hui.

Or si c'est bien l'idéologie qui sous-tend l'école aujourd'hui, Charlot remarque que «le principe du "chacun pour soi et en concurrence avec les autres" peut être en effet posé par la position libérale comme fait anthropologique, mais qu'il ne peut pas être soutenu comme valeur ». L'école de la compétition n'est appuyée sur aucune légitimation.

Que penser dès lors des propositions des neurosciences ou de la cyberculture : va-t-on y trouver une théorie qui pourrait armer une définition nouvelle et satisfaisante du projet éducatif humain?

8 Ce n'est pas le cas des neurosciences, selon Charlot: il leur reproche en effet de ne pas inscrire les apprentissages de l'élève dans leur réalité d'« un réseau de communications avec un professeur, des collègues, des parents, des frères et sœurs, etc., plus amplement, dans un certain rapport au savoir, au monde, aux autres et à lui-même ». Il parle même de discours d'intimidation par les neurosciences, avec recours à des formulations abusivement techniques, "pour mettre l'interlocuteur en position de faiblesse et lui faire gober n'importe quoi ».

9 De la culture numérique, il craint qu'elle se ramène à un " universel indéterminé et vide », «sans contenu particulier ». Et il redoute alors que par le numérique « les désirs se rencontrent et s'affrontent [...] sans cette médiation par l'autre qui constitue la culture » et qu'alors « menace la barbarie ».

Dans les deux cas au fond, la lanterne de Charlot ne lui permet pas de trouver une vision convaincante de l'éducation : il y voit ce travers commun qui consiste à « limiter l'homme à ses processus cérébraux et à un communicant en réseaux, qui peut dire ce qu'il veut parce qu'au fond ce n'est pas important».

11 Voici donc le passage dramatique du livre : on ne dispose plus de théorie pédagogique ni de l'éducation de rechange de celles du passé, puisque ni l'idéologie néo-libérale, ni le regard des neurosciences, ni l'idée de cyberculture n'assurent véritablement de sens à l'éducation.

Si l'on suit ce raisonnement qui interpelle, on a donc besoin d'une nouvelle définition de l'homme qui permette de définir ce qu'est l'éducation. L'auteur consacre alors de longs développements courageux à rassembler ce qu'on sait sur Sapiens, puis il soumet à son regard critique un certain nombre d'anthropologies récentes ou plus anciennes, pour chercher rien de moins qu'à identifier « les traits les plus durables de la condition humaine ", mais il souhaite le faire sans référence transcendante, sans deus ex machina. 

diversité, Charlot se demande alors quel est l'intérêt de recherches anthropologiques renvoyant à cette seule diversité au point qu'elles «ne disent au fond plus rien sur l'Homme ». Il est plutôt en accord avec Descola, quand il le résume en disant que la spécificité de l'espèce humaine "est produite au cours de l'évolution de l'espèce humaine et sous des formes particulières liées à l'histoire des groupes humains, de telle sorte que, tout à la fois, on ne la rencontre que chez l'homme, mais toujours sous des formes particulières ". Schaeffer s'oppose à toute idée d'« exception humaine » (la conscience elle-même étant «fabriquée par l'évolution » tout comme l'écholocation fait partie de la chauve-souris), Charlot accorde qu'en effet «nous ne parlerions pas sans les aires de Broca », mais il s'empresse d'ajouter que nous ne parlerions pas non plus «si nous n'avions pas quelque chose à dire à d'autres êtres humains ».

Et c'est bien non pas le langage lui-même dont l'homme n'a pas le monopole, mais sa culture, si développée et en développement constant, ce «moyen non génétique pour faire circuler de l'information entre individus ", qui définit l'humain. Or abordant et citant d'autres auteurs (Heidegger, Arendt), Charlot remarque avec de plus en plus de déception que ces thèses anthropologiques ne s'intéressent en aucune façon à l'enfant et son éducation. Ce qu'il appelle "le silence anthropo-pédagogique» lui semble regrettable.

Et c'est bien l'existence de cette culture, tellement spécifique à définir l'homme et son monde, qui requiert un mode aussi spécifique de transmission : l'éducation. L'éducation qui devient un véritable « droit anthropologique », devant assurer :

1. une humanisation: enseigner que l'espèce humaine est une aventure et que l'avenir de l'homme et de la planète est sous notre responsabilité ;

2. une socialisation-enculturation: enseigner l'unité génétique de l'espèce humaine et la diversité historique de ses modes culturels d'existence ;

3. une singularisation: enseigner la conscience de la liberté et de la dignité de chaque être humain.

On pose la lanterne. On a rarement lu d'explicitation des finalités fondamentales de l'éducation aussi fondée philosophiquement mais aussi susceptible de faire concrètement sens pour les divers systèmes d'éducation. Pourtant ce livre qui va si loin, qu'aucune question d'anthropologie philosophique ne semble rebuter et qui est obsédé par un passage qu'il appelle de ses vœux entre anthropologie et pédagogie, ce livre si ambitieux est en cela même un échec : en effet, quand il se risque, après avoir si bien cheminé, à esquisser quelques pistes pédagogiques, elles ne sont que d'une banalité déprimante. Le lecteur de cet ouvrage éminent reste sur sa faim, mais il a désormais, grâce au faisceau autour de la lanterne déposée, les éléments pour écrire la suite. 


\section{AUTEURS}

\section{ROGER-FRANÇOIS GAUTHIER}

Roger-François Gauthier, agrégé de lettres classiques et ancien élève de l'École nationale d'administration, est inspecteur général honoraire. Docteur en sciences de l'éducation, il est membre du comité de rédaction de la Revue internationale d'éducation de Sèvres. Délégué général du Comité universitaire d'information pédagogique (CUIP), il anime un collectif de recherche sur la question du curriculum. Courriel : erefgauthier[at]gmail.com 